\title{
ON THE INVISCID AND NON-RESISTIVE LIMIT FOR THE EQUATIONS OF INCOMPRESSIBLE MAGNETOHYDRODYNAMICS
}

\author{
J.I. DIAZ \\ Dept. Matemática Aplicada, Fac. CC. Matemáticas, Universidad Complutense de Madrid, \\ 28040 Madrid, Spain. \\ M.B. LERENA \\ Dept. Análisis Económico: Economía Cuantitativa, Fac. CC. Económicas y Empresariales, \\ Universidad Autónoma de Madrid, 28049, Madrid, Spain.
}

\begin{abstract}
We prove the convergence of the solutions for the incompressible homogeneous magnetohydrodynamics (MHD) system to the solutions to ideal MHD one in the inviscid and non-resistive limit, detailing the explicit convergence rates. For this study we consider a fluid occupying the whole space $\mathbb{R}^{3}$ and we assume that the viscosity effects in this fluid can be described by two different operators: the usual Laplacian operator affected by the inverse of the Reynolds number or by a viscosity operator introduced by S.I. Braginskii in 1965 .
\end{abstract}

\section{Introduction}

The magnetohydrodynamic (MHD) equations govern the macroscopic behavior of electrically conducting fluids submitted to the influence of magnetic fields and they are obtained by coupling the Navier-Stokes system for the fluid flow and the quasi-stationary Maxwell's equations (see, for instance, Cowling ${ }^{9}$ or Strhmer ${ }^{24}$ ).

The nondimensional form of the one-fluid homogeneous incompressible MHD system in the whole space $\mathbb{R}^{3}$ is given by

$$
\begin{cases}\frac{\partial \mathbf{u}}{\partial t}+(\mathbf{u} \cdot \nabla) \mathbf{u}-\mathbf{F}(\mathbf{u})+\nabla p+S \nabla\left(\frac{1}{2} \mathbf{B}^{2}\right)-S(\mathbf{B} \cdot \nabla) \mathbf{B}=\mathbf{f} & \text { in }(0, T) \times \mathbb{R}^{3}, \\ \frac{\partial \mathbf{B}}{\partial t}+(\mathbf{u} \cdot \nabla) \mathbf{B}-(\mathbf{B} \cdot \nabla) \mathbf{u}+\nu_{m} \operatorname{rot}(\operatorname{rot} \mathbf{B})=0 & \text { in }(0, T) \times \mathbb{R}^{3}, \\ \operatorname{div} \mathbf{u}=\mathbf{0} & \text { in }(0, T) \times \mathbb{R}^{3}, \\ \operatorname{div} \mathbf{B}=\mathbf{0} & \text { in }(0, T) \times \mathbb{R}^{3}, \\ \mathbf{u}(0, x)=\mathbf{u}_{0}(x) & \text { on } \mathbb{R}^{3}, \\ \mathbf{B}(0, x)=\mathbf{B}_{0}(x) & \text { on } \mathbb{R}^{3},\end{cases}
$$

for a fixed $T>0$, where $\mathbf{u}=\left(u_{i}\right)_{i=1,2,3}$ is the fluid velocity, $\mathbf{B}=\left(B_{i}\right)_{i}, i=$ 
$1,2,3$, the magnetic field, $p$ the pressure of the fluid and $\mathbf{f} \in \mathbb{R}^{3}$ represents a (nondimensional) volume density force. The two dimensionless numbers appearing in (1.1) are the magnetic viscosity $\nu_{m}:=\frac{1}{\mathrm{Rm}}\left(\right.$ with $\mathrm{Rm}:=L_{*} U_{*} \sigma \mu$ being the magnetic Reynolds number, which is proportional to the magnetic permeability $\mu$ and the electric conductivity of the fluid $\sigma$, being $L_{*}$ and $U_{*}$ the characteristic magnitudes for length and velocity) and the constant $S$ which is proportional to $\mu$. We have denote by $\mathbf{F}(\mathbf{u})$ the viscosity forces acting on the flow which will be specified later on.

When in the above system we neglect the viscosity forces in the fluid and we assume it to be perfectly conductor, i.e., non-resistive, we are under the hypothesis of ideal magnetohydrodynamics whose mathematical equations are given by

$$
\begin{cases}\frac{\partial \mathbf{u}^{0}}{\partial t}+\left(\mathbf{u}^{0} \cdot \nabla\right) \mathbf{u}^{0}+\frac{S}{2} \nabla\left(\mathbf{B}^{0}\right)^{2}-S\left(\mathbf{B}^{0} \cdot \nabla\right) \mathbf{B}^{0}+\nabla p^{0}=\mathbf{f} & \text { in }(0, T) \times \mathbb{R}^{3}, \\ \frac{\partial \mathbf{B}^{0}}{\partial t}+\left(\mathbf{u}^{0} \cdot \nabla\right) \mathbf{B}^{0}-\left(\mathbf{B}^{0} \cdot \nabla\right) \mathbf{u}^{0}=0 & \text { in }(0, T) \times \mathbb{R}^{3}, \\ \operatorname{div} \mathbf{u}^{0}=\mathbf{0} & \text { in }(0, T) \times \mathbb{R}^{3}, \\ \operatorname{div} \mathbf{B}^{0}=\mathbf{0} & \text { in }(0, T) \times \mathbb{R}^{3}, \\ \mathbf{u}^{0}(0, x)=\mathbf{u}_{0}^{0}(x) & \text { on } \mathbb{R}^{3}, \\ \mathbf{B}^{0}(0, x)=\mathbf{B}_{0}^{0}(x) & \text { on } \mathbb{R}^{3},\end{cases}
$$

The MHD systems appear in diverse areas of interest, being the base of many complex models in astrophysics and nuclear fusion theory (see, for example, Biskamp $^{2}$, Freidberg ${ }^{13}$ ). We are specially interested in this last application, where the incompressible ideal MHD plays an important role, providing the simplest model for the detection and description of equilibrium and stability properties for a magnetically confined plasma in fusion reactors as Tokamaks or Stellarators (see, e.g., Braginskii ${ }^{3}$, Freidberg ${ }^{13}$ or Hazeltine and Meiss ${ }^{15}$ ). In these reactors, there are gases in plasma state, whose behavior is modelled by the ideal MHD model, coexisting with other gases that are not under the hypothesis of ideality.

In the present paper we are concerned with the transition from MHD to ideal MHD in the whole space $\mathbb{R}^{3}$. Mathematically, this study is equivalent to establish the convergence of the solutions of the MHD system (1.1) to solutions of the ideal one (1.2), in the limit to infinity of the electric conductivity $\sigma$ and to zero of the viscosity operator $\mathbf{F}$ (the rigorous meaning of this limit will be cleared up once we have detailed $\mathbf{F}$ ). We point out the analogy between this convergence and the passing from the Navier-Stokes equations to the Euler's one in fluid dynamic, for which an extensive bibliography can be found since the pioneer works of Kato ${ }^{17}$ or Swann ${ }^{26}$. In the study that we develop here, we will suppose that the viscosity effects in the 
fluid are described by the usual Laplacian operator affected by the inverse of the Reynold's number Re. But we shall also consider the case where viscosity is described by the Braginskii's operator, introduced by I.S. Braginskii in $1965,{ }^{3}$ which is widely used in the theory of fusion plasmas by magnetic confinement (see, e.g., Hazeltine and Meiss ${ }^{15}$ ).

We have organized this article as follows: in Section 2 we introduce the functional framework in which we shall work and we present the operators that we shall use for describing the viscosity forces in the fluid; in Section 3 we give a local in time existence and uniqueness result for the MHD systems which we shall use for the setting of the convergence of the solutions. Finally, in Section 4 we state the convergence for both viscosity operators in the norms of the Lebesgue space $L^{2}$ and in the Sobolev spaces $W^{2, s}$ for $s>\frac{3}{2}$, giving explicit convergence rates. In this direction, we must mention the existence of two related papers due to $\mathrm{Wu}^{31}$ and Díaz ${ }^{11}$, where the authors study, by using independent arguments, the convergence of the solution of the MHD system, with $\mathbf{F}=\frac{1}{\mathbf{R e}} \Delta$, in the limit $\mathbf{R e}, \operatorname{Rm} \rightarrow+\infty$ and $S \rightarrow 0$. This last limit, which is explicitly used in their proofs, is related with the degree of capacity of fluids flows to be shaped by using magnetic fields.

\section{The Viscosity Operators}

Due to the homogeneous incompressible character of the fluids that we are dealing with, the natural framework in which we shall work is that of the solenoidal vector fields of $L^{2}\left(\mathbb{R}^{3}\right)^{3}$. We shall denote by $H^{s}$ the vectorial $L^{2}$ type Sobolev spaces $H^{s}\left(\mathbb{R}^{3}\right)^{3}=W^{s, 2}\left(\mathbb{R}^{3}\right)^{3}$, with $s \geq 0$ (not necessarily an integer) and we shall use $($,$) and (,)_{s}$ for the inner product in $L^{2}$ and $H^{s}$ respectively; \|\|$_{0}$ and \|\|$_{s}$ will be used for the associated norms in those spaces (throughout this paper we shall use this notation indiscriminately for scalars and vectors).

Let $P$ be the Leray operator, which maps vector-valued function into divergence-free vectors, given by $P_{j k}=\delta_{j k}-R_{j} R_{k}, j, k=1,2,3$, where $R_{j}$ are the Riesz transforms. It is well known (see, e.g., Constantin and Foias ${ }^{8}$, Kato and Ponce ${ }^{19}$ or Cannone ${ }^{6}$ ) that this projection it is a bounded operator acting on $L^{2}\left(\mathbb{R}^{3}\right)^{3}$ and $H^{s}, s>0$. Throughout this paper we shall denote by $H_{\sigma}^{s}$ the image by $P$ of the $H^{s}$ spaces with $s>0$ and by $H_{\sigma}=P L^{2}\left(\mathbb{R}^{3}\right)^{3}$. That is,

$$
H_{\sigma}^{s}=\left\{\mathbf{u} \in H^{s}: \operatorname{div} \mathbf{u}=0\right\} .
$$

Next, let us describe the viscosity force $\mathbf{F}$ that appears in (1.1). As we mentioned in the Introduction, we shall consider viscosity effects described by two different operators. Usually, in an incompressible liquid these effects 
are assumed to be given by

$$
\mathbf{F}(\mathbf{u})=\frac{1}{\mathbf{R e}} \Delta \mathbf{u}
$$

where $\mathbf{R e}:=\frac{L_{*} U_{*}}{\nu}$ is the Reynolds number and $\nu$ the kinematic viscosity of the fluid. In this paper we shall also contemplate the situation in which viscosity is described by the Braginskii operator $\mathbf{F}(\mathbf{u})=\widehat{V} \mathbf{u}\left(\right.$ see Braginskii $\left.{ }^{3}\right)$, that appears very often in the study of fusion plasmas by magnetic confinement (see, for instance, Freidberg ${ }^{13}$, Hazeltine and Meiss ${ }^{15}$ ). This operator is given by

$$
(\widehat{V} \mathbf{u})_{i}=\frac{-\partial \pi_{i j}}{\partial x_{j}}, i, j=1,2,3
$$

(summation under repeated index is understood), where $\pi_{i j}$ is the stress tensor defined by mean of five viscosity coefficients $\mu_{\alpha}, \alpha=0,1, \ldots, 4$, as:

$$
\pi_{i j}=\sum_{\alpha=0}^{4} \gamma_{\alpha} \mu_{\alpha} W_{\alpha i j}\left(\gamma_{\alpha}=-1 \text { if } \alpha=0,1,2 \text { and } \gamma_{\alpha}=1 \text { for } \alpha=3,4\right),
$$

with $W_{\alpha i j}:=\sum_{k, l=0}^{3} A_{\alpha i j, k l}(\mathbf{h}) W_{k l}$ and $W_{k l}=\frac{\partial u_{k}}{\partial x_{l}}+\frac{\partial u_{l}}{\partial x_{k}}-\frac{2}{3} \delta_{k l} \nabla \cdot \mathbf{u}$. The coefficients $A_{\alpha i j, k l}$ are polynomials in $\mathbf{h}:=\mathbf{B} /|\mathbf{B}|$ and are given on page 250 of Braginskii, ${ }^{3}$ while the viscosity coefficients $\mu_{\alpha}(\alpha=0,1, \ldots, 4)$ are positive and just depend on $|\mathbf{B}|$. Nevertheless, in order to avoid this pathological situation, we shall use here the approach already followed by Spada and Wobig $^{23}$ (see also ${ }^{3}$ for a slightly different approach) where the coefficients in $\widehat{V}$ result to be independent of the magnetic field (that is, $A_{\alpha i j, k l}$ are constants). In particular, we shall assume that the viscosity coefficients $\mu_{\alpha}$ are approximated by positive constants.

It is well known (see, e.g., Constantin and Foias ${ }^{8}$ ) that, in the absence of boundaries, the Laplacian operator $-\Delta$ and the Stokes's one $-P \Delta$ acting on the $H^{s}$-spaces are identical (that is, $-\Delta$ and the projection $P$ commute). It is easy to check, by using the Fourier transform, that, under the above hypothesis on $\mu_{\alpha}$ and $A_{\alpha i j, k l}$, this commutation also holds for the Braginskii operator $\widehat{V}$. Furthermore, we have the following property:

Lemma 2.1. $\left(-\widehat{V}, H_{\sigma}^{2}\right)$ is a continuous and m-dissipative operator acting on $H_{\sigma}$.

Proof. In order to prove that $\left(-\widehat{V}, H_{\sigma}^{2}\right)$ is a m-dissipative operator in $H_{\sigma}$ we use the bilinear form $\mathcal{E}: H^{1}\left(\mathbb{R}^{3}\right)^{3} \times H^{1}\left(\mathbb{R}^{3}\right)^{3} \longrightarrow \mathbb{R}$ introduced by Spada and Wobig, ${ }^{23}$

$$
\mathcal{E}(\mathbf{u}, \mathbf{v})=\sum_{\alpha=0}^{4} \gamma_{\alpha} \mu_{\alpha} \int_{\mathbb{R}^{3}}\left(A_{\alpha i j k l}+A_{\alpha i j l k}\right) \frac{\partial u_{k}}{\partial x_{l}} \frac{\partial v_{i}}{\partial x_{j}} d \mathbf{x} .
$$


This form is continuous and coercive and verifies (see Spada and Wobig ${ }^{23}$ )

$$
\mathcal{E}(\mathbf{u}, \mathbf{v})=(-\widehat{V} \mathbf{u}, \mathbf{v}), \forall \mathbf{u}, \mathbf{v} \in H_{\sigma}^{1}
$$

Then, $\left(-\widehat{V}, H_{\sigma}^{2}\right)$ is dissipative in $H_{\sigma}$; in particular $\left(\mathrm{see}^{23}\right)$

$$
(-\widehat{V} \mathbf{v}, \mathbf{v})=\mathcal{E}(\mathbf{v}, \mathbf{v}) \geq \min _{\alpha}\left(\mu_{\alpha}\right)\|\nabla \mathbf{v}\|_{0}^{2} \geq 0 .
$$

Thus, if we prove there exists $\lambda_{0}>0$ such that, $\forall \mathbf{g} \in H_{\sigma}^{2}$ there exists $\mathbf{v} \in H_{\sigma}^{2}=D(\widehat{V})$ being the unique solution to $\mathbf{v}-\lambda_{0} \widehat{V} \mathbf{v}=\mathbf{g}$, we would have that $\left(-\widehat{V}, H_{\sigma}^{2}\right)$ is m-dissipative (see, e.g., Cazenave and Haraux ${ }^{7}$ ). Indeed, we consider the restriction of $\mathcal{E}$ to the divergence-free space $H_{\sigma}^{1} \times H_{\sigma}^{1}$ and we define a new bilinear form a : $H_{\sigma}^{1} \times H_{\sigma}^{1} \longrightarrow \mathbb{R}$

$$
\mathrm{a}(\mathbf{v}, \mathbf{w}):=\mathcal{E}(\mathbf{v}, \mathbf{w})+(\mathbf{v}, \mathbf{w}) .
$$

This bilinear form is again continuous and coercive, hence we can appeal to Lax-Milgram's theorem to conclude that there exists an unique $\mathbf{v} \in$ $L^{2}\left(\mathbb{R}^{3}\right)^{3}$ such that

$$
\mathbf{v}-\widehat{V} \mathbf{v}=\mathbf{g}
$$

Moreover, by elliptic-regularity results (see, for example, ${ }^{14}$ ) and using that the projection $P$ commutes with Braginskii's operator, we deduce that $\mathbf{v} \in$ $H_{\sigma}^{2}$.

For the continuity of $\widehat{V}$ in $H_{\sigma}$, we use that $\forall \mathbf{v} \in H_{\sigma}^{2}$,

$$
(\widehat{V} \mathbf{v})_{i}=-\sum_{\alpha=0}^{4} \gamma_{\alpha} \mu_{\alpha} \int_{\mathbb{R}^{3}} A_{\alpha i j k l}\left(\frac{\partial^{2} v_{k}}{\partial x_{j} \partial x_{l}}+\frac{\partial^{2} v_{l}}{\partial x_{j} \partial x_{k}}\right) d \mathbf{x}, i=1,2,3 .
$$

Hence, as $\left|\gamma_{\alpha}\right|=1$,

$$
\begin{gathered}
\left\|(\widehat{V} \mathbf{v})_{i}\right\|_{0}^{2}=\int_{\mathbb{R}^{3}}\left\{\sum_{k, l, j=1}^{3}\left[-\sum_{\alpha=0}^{4} \gamma_{\alpha} \mu_{\alpha} A_{\alpha i j k l}\left(\frac{\partial^{2} v_{k}}{\partial x_{j} \partial x_{l}}+\frac{\partial^{2} v_{l}}{\partial x_{j} \partial x_{k}}\right)\right]\right\}^{2} d \mathbf{x} \leq \\
\leq\left(4\left(\max _{\alpha, j, k, l, i}\left|A_{\alpha i j k l}\right|\right)\left(\max _{\alpha} \mu_{\alpha}\right)\right)^{2} \int_{\mathbb{R}^{3}}\left\{\sum_{k, l, j=1}^{3}\left|\frac{\partial^{2} v_{k}}{\partial x_{j} \partial x_{l}}+\frac{\partial^{2} v_{l}}{\partial x_{j} \partial x_{k}}\right|\right\}^{2} d \mathbf{x}
\end{gathered}
$$

$\leq k^{\prime}\left(\max _{\alpha} \mu_{\alpha}\right)^{2}\|\mathbf{v}\|_{2}^{2}$. 
with $k^{\prime}:=\left(4 \max _{\alpha, j, k, l, i}\left|A_{\alpha i j k l}\right|\right)^{2}$.

Remark 2.2. From the above proof, it follows that $\widehat{V}$ can be also consider as a continuous operator acting on $H_{\sigma}^{m}$ into $H_{\sigma}^{m-2}$, i.e., there exists $k^{\prime \prime}>0$ constant such that

$$
\|\widehat{V} \mathbf{v}\|_{m-2} \leq k^{\prime \prime}\left(\max _{\alpha=0, \ldots, 4} \mu_{\alpha}\right)\|\mathbf{v}\|_{m}, \mathbf{v} \in H_{\sigma}^{m}\left(\mathbb{R}^{3}\right)^{3}, m \geq 2 .
$$

\section{Existence Results}

The existence and uniqueness of solutions for both the MHD and ideal MHD systems have been already proved in previous papers, when the viscosity term in (1.1) is taken to be (2.3). In particular, we shall mention the works by Duvaut and Lions ${ }^{12}$ and Sermange and Temam ${ }^{28}$ for the MHD system in a bounded domain or in the whole spaces $\mathbb{R}^{n}, n=2,3$, with periodic boundary conditions; for the ideal case, we mention the articles by Schmidt ${ }^{21}$ and $\mathrm{Secchi}^{22}$ where the existence and uniqueness of local strong solutions is proved in a bounded domain, and the paper by Sulem ${ }^{25}$ for the whole $3 \mathrm{~d}$-space with analytic initial data. Concerning the viscosity operator of Braginskii (2.4), Spada and Wobig ${ }^{23}$ proved the existence and uniqueness of a weak solution for the stationary MHD system in a bounded domain.

In this paper, we give a local existence and uniqueness result in the spaces $H_{\sigma}^{s}$ above introduced, for the ideal MHD and MHD systems, for both (2.3) and (2.4) viscosity operators (for which, from now on, we shall use the notation $\mathbf{F}$ ), whose proof lies on some results for evolution abstracts equation due to Kato $\left(\mathrm{see}^{16},{ }^{19}\right)$. In order to apply this theory to the MHD systems we shall introduce some operators defined on the divergence-free spaces $H_{\sigma}$. We start with the linear operator $\mathcal{A}_{1}$ defined on $H_{\sigma} \times H_{\sigma}$ as

$$
\mathcal{A}_{1}=\left(\begin{array}{cc}
-\mathbf{F} & 0 \\
0 & -\frac{1}{\mathrm{Rm}} \Delta
\end{array}\right),
$$

with domain $D\left(\mathcal{A}_{1}\right)=H_{\sigma}^{2} \times H_{\sigma}^{2}$ (when $\mathbf{F}=\frac{1}{R_{e}} \Delta$ this is a well known result and in the case of $\mathbf{F}=\widehat{V}$ it can be easily checked by using standard elliptic regularity arguments (see, e.g., $\left.{ }^{14}\right)$ ).

Let be $\boldsymbol{\Phi}=(\mathbf{u}, \mathbf{B}) \in H_{\sigma}^{r} \times H_{\sigma}^{r}, r>\frac{3}{2}$, fixed. We define another linear operator on $H_{\sigma} \times H_{\sigma}$ by

$$
\mathcal{A}_{2}(\Phi)=\left(\begin{array}{cc}
P(\mathbf{u} \cdot \nabla) & -S P(\mathbf{B} \cdot \nabla) \\
-P(\mathbf{B} \cdot \nabla) & P(\mathbf{u} \cdot \nabla)
\end{array}\right)
$$

with $D\left(\mathcal{A}_{2}(\Phi)\right)=H_{\sigma}^{1} \times H_{\sigma}^{1}$ (notice that, from Sobolev's imbedding $H_{\sigma}^{r} \hookrightarrow$ $\left(L^{\infty}\left(\mathbb{R}^{3}\right)\right)^{3}$ when $r>\frac{3}{2}$, and so $(\mathbf{u} \cdot \nabla) \mathbf{v} \in\left(L^{2}\left(\mathbb{R}^{3}\right)\right)^{3}$ for every $\left.\mathbf{v} \in H_{\sigma}^{1}\right)$. 
Finally, let us denote by $(\mathcal{A}(\boldsymbol{\Phi}), D(\mathcal{A}(\boldsymbol{\Phi})))$ the operator sum of the two above introduced, i.e.,

$$
\mathcal{A}(\boldsymbol{\Phi})=\mathcal{A}_{1}+\mathcal{A}_{2}(\boldsymbol{\Phi}), D(\mathcal{A}(\boldsymbol{\Phi}))=H_{\sigma}^{2} \times H_{\sigma}^{2} .
$$

We get that

Lemma 3.1. Let $\mathbf{\Phi} \in H_{\sigma}^{r} \times H_{\sigma}^{r}, r>\frac{3}{2}$, be fixed. Then, $(-\mathcal{A}(\mathbf{\Phi}), D(\mathcal{A}(\mathbf{\Phi})))$ generates a contraction semigroup in $H_{\sigma} \times H_{\sigma}$.

Proof. Let us first note that, as we are working with solenoidal vector fields, the following identity holds:

$$
((\mathbf{u} \cdot \nabla) \mathbf{v}, \mathbf{v})=0, \forall \mathbf{u} \in H_{\sigma}^{r}, \forall \mathbf{v} \in H_{\sigma}^{1} .
$$

Thus, given $\mathbf{\Phi} \in H_{\sigma}^{r} \times H_{\sigma}^{r}$,

$$
\left(\mathcal{A}_{2}(\mathbf{\Phi}) \Psi, \Psi\right)=0, \forall \mathbf{\Psi} \in H_{\sigma}^{1} \times H_{\sigma}^{1}
$$

and so $\left(-\mathcal{A}_{2}(\boldsymbol{\Phi}), H_{\sigma}^{1} \times H_{\sigma}^{1}\right)$ is dissipative. Moreover, using that $P$ is an unitary operator on the $H^{s}$-spaces and from Sobolev's imbedding, it follows the existence of a positive constant $c$ such that

$$
\begin{aligned}
\left\|\mathcal{A}_{2}(\mathbf{\Phi}) \mathbf{\Psi}\right\|_{0}= & \|P(\mathbf{u} \cdot \nabla) \mathbf{v}-S P(\mathbf{B} \cdot \nabla) \mathbf{C}\|_{0}+\|P(\mathbf{u} \cdot \nabla) \mathbf{C}-P(\mathbf{B} \cdot \nabla) \mathbf{v}\|_{0} \\
& \leq c\|\mathbf{v}\|_{1}\left(\|\mathbf{u}\|_{r}+\|\mathbf{B}\|_{r}\right)+c\|\mathbf{C}\|_{1}\left(\|\mathbf{u}\|_{r}+S\|\mathbf{B}\|_{r}\right) \leq \\
& \leq c \max (1, S)\|\mathbf{\Phi}\|_{r}\|\mathbf{\Psi}\|_{2}=k\|\mathbf{\Psi}\|_{2} \forall \mathbf{\Psi}=(\mathbf{v}, \mathbf{C}) \in H_{\sigma}^{2} \times H_{\sigma}^{2}
\end{aligned}
$$

which allows us to state that $\left(\mathcal{A}_{2}(\boldsymbol{\Phi}), D\left(\mathcal{A}_{2}(\boldsymbol{\Phi})\right)\right)$ it is $\mathcal{A}_{1}$-bounded with relative bound being 0 .

Therefore, if we prove that $\left(\mathcal{A}_{1}, D\left(\mathcal{A}_{1}\right)\right)$ generates a contraction semigroup in $H_{\sigma} \times H_{\sigma}$, we can appeal to the theory of perturbed operators (see, for example, ${ }^{18}$ or $\left.^{27}\right)$ and conclude that $\left(-\mathcal{A}(\boldsymbol{\Phi}), H_{\sigma}^{2} \times H_{\sigma}^{2}\right)$ is the infinitesimal generator of a contraction semigroup in $H_{\sigma} \times H_{\sigma}$. But when $\mathbf{F}=\frac{1}{\mathbf{R e}} \Delta$, it is a well-known result (see, for instance, $\left.{ }^{7}\right)$ that $\left(-\mathcal{A}_{1}, D\left(\mathcal{A}_{1}\right)\right)$ generates a contraction semigroup on $H_{\sigma} \times H_{\sigma}$ given by $S(t)=\left(\mathrm{e}^{\frac{1}{\mathrm{Re}} \Delta t}, \mathrm{e}^{\frac{1}{\mathrm{Rm}} \Delta t}\right)$ where $\mathrm{e}^{\Delta t}$ is the heat semigroup. In case that $\mathbf{F}=\widehat{V}$, we use that $\left(-\widehat{V}, H_{\sigma}^{2}\right)$ is a mdissipative operator on $H_{\sigma}$ (see Lemma 2.1) and from Hille-Yosida-Phillips's theorem (see, e.g. ${ }^{7}$ ), we get that this operator also generates a contraction semigroup on $H_{\sigma}$.

Let $\mathrm{S}$ be the isometric isomorphism $\mathrm{S}:=(I-\Delta)^{1 / 2}: H^{r} \longrightarrow H^{r-1}$, $r \geq 1$, that appears when defining the Sobolev spaces of exponent 2 via the Fourier transform (see, e.g. ${ }^{20}$ ). We can consider the restriction of this operator to the divergence-free spaces, that we shall also denote by S, i.e.,

$$
\mathrm{S}^{r}=(I-\Delta)^{r / 2}: H_{\sigma}^{r} \longrightarrow H_{\sigma}, r \geq 1
$$


that it is also an isometry (note that the expression of $\mathrm{S}^{r}$ in the Fourier variables is $\left(1+|\xi|^{2}\right)$ which commute with the unitary projection $\left.P\right)$. We denote by $\Lambda^{r}$ the product operator of components $\Lambda^{r}=\left(\mathrm{S}^{r}, \mathrm{~S}^{r}\right)$, i.e., $\Lambda^{r}$ : $H_{\sigma}^{r} \times H_{\sigma}^{r} \longrightarrow H_{\sigma} \times H_{\sigma}$, which verifies the following lemma:

Lemma 3.2. Let be $\boldsymbol{\Phi} \in H_{\sigma}^{r} \times H_{\sigma}^{r}, r>\frac{5}{2}$ fixed. There exits a positive constant $\lambda$, just depending on $\boldsymbol{\Phi}$, such that

$$
\left\|\left[\Lambda^{r}, \mathcal{A}(\mathbf{\Phi})\right] \Lambda^{-r}\right\|_{\mathcal{L}\left(H_{\sigma} \times H_{\sigma}\right)} \leq \lambda
$$

where [,] denotes the commutator operator.

Remark 3.3. The above lemma it is formally equivalent $\left(\mathrm{see}^{16} \mathrm{or}^{19}\right)$ to the existence of a bounded linear operator $B(\boldsymbol{\Phi}) \in \mathcal{L}\left(H_{\sigma} \times H_{\sigma}\right)$ such that $\Lambda^{s} \mathcal{A}(\boldsymbol{\Phi}) \Lambda^{-s}=\mathcal{A}(\boldsymbol{\Phi})+B(\boldsymbol{\Phi})$. We also note, that the same result also holds when we consider not the operator $\mathcal{A}(\boldsymbol{\Phi})$ but $\mathcal{A}_{2}(\boldsymbol{\Phi})$.

Proof. We recall the estimate

$$
\left\|\left[(I-\Delta)^{r / 2},(\mathbf{u} \cdot \nabla)\right](I-\Delta)^{-r / 2}\right\|_{0} \leq c\|\nabla \mathbf{u}\|_{r-1}, \text { for a fixed } \mathbf{u} \in H_{\sigma}^{r}
$$

obtained in ${ }^{16}$ for the study of the Navier-Stokes equations. Due to the structure of the operator $\mathcal{A}(\boldsymbol{\Phi})$ it suffices to show that an estimate of type

$$
\left\|\left[\mathrm{S}^{r}, \mathbf{F}\right] \mathrm{S}^{-r}\right\|_{0} \leq \lambda
$$

holds for the viscosity operator $\mathbf{F}$, where $\lambda$ is positive constant. For this purpose we use that their expressions in the Fourier variables are $\mathcal{F}(\mathbf{F})(\xi)=$ $p(\xi)$ for a.e. $\xi \in \mathbb{R}^{3}$ (we have denoted by $\mathcal{F}$ the Fourier transform), where $p(\xi)=|\xi|^{2}$ when $\mathbf{F}=\Delta$ and $p$ is a suitable more complicated second order polynomial in the variable $\xi$ in the case of the Braginskii operator (2.4) Thus, by using Parseval's identity, we obtain that

$$
\begin{gathered}
\left\|\left[(I-\Delta)^{r / 2}, \mathbf{F}\right](I-\Delta)^{-r / 2} \mathbf{v}\right\|_{0}=\left\|\mathcal{F}\left(\left[(I-\Delta)^{r / 2}, \mathbf{F}\right](I-\Delta)^{-r / 2} \mathbf{v}\right)\right\|_{0} \\
=\left\|\left[\left(1+|\xi|^{2}\right)^{r / 2} p(\xi)\right]\left(1+|\xi|^{2}\right)^{-r / 2} \widehat{\mathbf{v}}-p(\xi)\left(1+|\xi|^{2}\right)^{r / 2}\left(1+|\xi|^{2}\right)^{-r / 2} \widehat{\mathbf{v}}\right\|_{0}=0
\end{gathered}
$$

which gives the desired result.

Finally, we can consider the operator $\mathcal{A}$ mapping $H_{\sigma}^{r} \times H_{\sigma}^{r}, r>\frac{3}{2}$, into the set of generators of contraction semigroups on $H_{\sigma} \times H_{\sigma},(-\mathcal{A}(\boldsymbol{\Phi}), D(\mathcal{A}(\mathbf{\Phi})))$. The restriction of this operator to the space $H_{\sigma}^{s} \times H_{\sigma}^{s}$ with $s>\frac{5}{2}$ satisfies the following Lipschitz property:

Lemma 3.4. There exists a positive constant $L$ such that:

$$
\|\mathcal{A}(\mathbf{\Phi})-\mathcal{A}(\mathbf{\Psi})\|_{\mathcal{L}\left(H_{\sigma}^{s} \times H_{\sigma}^{s}, H_{\sigma} \times H_{\sigma}\right)} \leq L\|\mathbf{\Phi}-\mathbf{\Psi}\|_{0},
$$


for every $\mathbf{\Phi}, \Psi \in H_{\sigma}^{s} \times H_{\sigma}^{s}$ with $s>\frac{5}{2}$.

Proof. We first note that, as we are assuming $s>\frac{5}{2}, H_{\sigma}^{s} \times H_{\sigma}^{s} \hookrightarrow H_{\sigma}^{2} \times H_{\sigma}^{2}$ and hence the above estimate makes sense. Due to the structure of the operator $\mathcal{A}$ (notice that $\mathcal{A}_{1}$ in the definition of $\mathcal{A}(\boldsymbol{\Phi})$ does not depend on $\boldsymbol{\Psi})$, the proof of this estimate reduces to show that

$$
\|(\mathbf{u} \cdot \nabla) \mathbf{w}-(\mathbf{v} \cdot \nabla) \mathbf{w}\|_{0} \leq L\|\mathbf{u}-\mathbf{v}\|_{0}\|\mathbf{w}\|_{s} \text { for every } \mathbf{u}, \mathbf{v}, \mathbf{w} \in H_{\sigma}^{s} .
$$

But this is straight consequence of Hölder's inequality and the Sobolev imbedding $H^{s-1} \hookrightarrow L^{\infty}\left(\mathbb{R}^{3}\right)^{3}$ for $s>\frac{5}{2}$. Indeed,

$$
\|(\mathbf{u} \cdot \nabla) \mathbf{w}-(\mathbf{v} \cdot \nabla) \mathbf{w}\|_{0} \leq\|\mathbf{u}-\mathbf{v}\|_{0}\|\nabla \mathbf{w}\|_{L^{\infty}} \leq c\|\mathbf{u}-\mathbf{v}\|_{0}\|\mathbf{w}\|_{s}
$$

where $c$ is the constant appearing in the Sobolev imbedding.

Remark 3.5. From the above lemma, we can deduce that for a given $\boldsymbol{\Phi} \in H_{\sigma}^{r} \times H_{\sigma}^{r}, r>\frac{3}{2}$, the restriction of the operator $(\mathcal{A}(\boldsymbol{\Phi}), D(\mathcal{A}(\mathbf{\Phi})))$ to the spaces $H_{\sigma}^{s} \times H_{\sigma}^{s}$ with $s>\frac{5}{2}$, it is a linear bounded operator. That is, $\left.\mathcal{A}(\mathbf{\Phi})\right|_{H_{\sigma}^{s} \times H_{\sigma}^{s}} \in \mathcal{L}\left(H_{\sigma}^{s} \times H_{\sigma}^{s}, H_{\sigma} \times H_{\sigma}\right)$.

We are now in position to state the existence and uniqueness result for the MHD systems. We begin with the viscous case:

Theorem 3.6. Let be $T>0$ and $\left(\mathbf{u}_{0}, \mathbf{B}_{0}\right) \in H^{s}\left(\mathbb{R}^{3}\right)^{3} \times H^{s}\left(\mathbb{R}^{3}\right)^{3}, s>\frac{5}{2}$, such that $\nabla \cdot \mathbf{u}_{0}=\nabla \cdot \mathbf{B}_{0}=0$. Let us assume that $\mathbf{f} \in \mathcal{C}\left([0, T] ; L^{2}\left(\mathbb{R}^{3}\right)\right)$ $\cap L^{1}\left(0, T ; H_{\sigma}^{s}\left(\mathbb{R}^{3}\right)\right)$. Then, there exists a couple $(\mathbf{u}, \mathbf{B})$ being the unique solution to (1.1), with $\mathbf{F}$ given by (2.3) or (2.4), satisfying

$$
(\mathbf{u}, \mathbf{B}) \in \mathcal{C}\left(\left[0, T^{\prime}\right] ;\left(H^{s}\left(\mathbb{R}^{3}\right)^{3}\right)^{2}\right) \cap \mathcal{C}^{1}\left(\left[0, T^{\prime}\right] ; H^{s-2}\left(\mathbb{R}^{3}\right)^{3} \times H^{s-2}\left(\mathbb{R}^{3}\right)^{3}\right)
$$

where $T^{\prime} \in(0, T]$ just depends on the initial data and f. Moreover, there exists a constant $C>0$, independent of the viscosities, such that

$$
\|\mathbf{u}(t)\|_{s}+\|\mathbf{B}(t)\|_{s} \leq C, \forall t \in\left[0, T^{\prime}\right] .
$$

Proof. In order to prove this theorem, we project the MHD system into the space of solenoidal vector fields of $L^{2}\left(\mathbb{R}^{3}\right)^{3}$ by means of the Leray operator. Then, using the operators that we have introduced all along this section, we can write the projected system as the Cauchy problem

$$
\left\{\begin{array}{l}
\frac{d \Phi}{d t}+\mathcal{A}(\Phi) \Phi=\mathbf{G}(t) \\
\Phi(0)=\left(\mathbf{u}_{0}, \mathbf{B}_{0}\right)
\end{array}\right.
$$


where $\boldsymbol{\Phi}$ takes value on the Hilbert space $H_{\sigma} \times H_{\sigma}$ and $\mathbf{G}(t):=\left(\begin{array}{l}\mathbf{f}(t) \\ 0\end{array}\right)$. Lemmas 3.1, 3.2 and 3.4 allow us to appeal to the theory developed in Kato ${ }^{16}$ (see also ${ }^{19}$ ) for abstract evolution equations and conclude the existence of an unique $\mathbf{\Phi}=(\mathbf{u}, \mathbf{B})$, solution of (3.10) satisfying

$$
\Phi \in \mathcal{C}\left(\left[0, T^{*}\right] ; H_{\sigma}^{s} \times H_{\sigma}^{s}\right) \cap \mathcal{C}^{1}\left(\left[0, T^{*}\right] ; H_{\sigma} \times H_{\sigma}\right)
$$

for some $T^{*} \in(0, T]$ which just depends on initial data (we note that the bounds in Lemmas 3.2 and 3.4 do not depend on the viscosities $\nu_{m}$, Re or $\left.\mu_{\alpha}\right)$.

These results by Kato ${ }^{16}$ prove the existence and uniqueness of local in time solutions for quasi-linear equations of type (3.10), where $\boldsymbol{\Phi}(t)$ takes values in a Banach space $X ; \mathcal{A}(\boldsymbol{\Phi})$ is a linear operator on $X$ and there exist a subspace $Y \hookrightarrow X$ dense, such that for every $\boldsymbol{\Phi} \in Y,(-\mathcal{A}(\boldsymbol{\Phi}), D(\mathcal{A}(\boldsymbol{\Phi})))$ generates a $\mathcal{C}^{0}$ semigroup on $X$. Roughly speaking, the proof of this result lies in showing the existence of a fixed point for the mapping $\boldsymbol{\Psi} \longrightarrow \boldsymbol{\Phi}$ where $\boldsymbol{\Phi}$ solves $\frac{d \boldsymbol{\Phi}}{d t}+\mathcal{A}(\boldsymbol{\Psi}) \boldsymbol{\Phi}=\mathbf{G}(t)$ with $\boldsymbol{\Phi}(0)=\left(\mathbf{u}_{0}, \mathbf{B}_{0}\right)$, by means of the contraction mapping theorem. To this end, the operator $\mathcal{A}$ must satisfy a Lipschitz condition similar to that of Lemma 3.4. In the case of the MHD system, we have chosen $H_{\sigma} \times H_{\sigma}$ to play the role of $X$ and space $Y$ is taken to be $H_{\sigma}^{s} \times H_{\sigma}^{s}$, where $s>\frac{5}{2}$ is the lowest exponent for which Lemma 3.4 holds.

The additional regularity on $\boldsymbol{\Phi}$, i.e., $\boldsymbol{\Phi} \in \mathcal{C}^{1}\left(\left[0, T^{*}\right], H_{\sigma}^{s-2} \times H_{\sigma}^{s-2}\right)$, is a consequence of

$$
\frac{d \Phi}{d t}(t)=-\mathcal{A}(\Phi(t)) \Phi(t)+F(t) \in H_{\sigma}^{s-2} \times H_{\sigma}^{s-2}, \quad \forall t \in\left[0, T^{*}\right]
$$

being a continuous function on $\left[0, T^{*}\right]$. Indeed, from the regularity in (3.11) we know that $\mathcal{A}_{1} \Phi \in \mathcal{C}\left(\left[0, T^{*}\right] ; H_{\sigma}^{s-2} \times H_{\sigma}^{s-2}\right)$; moreover, $H_{\sigma}^{s-1}$ is a Banach algebra for $s>\frac{5}{2}$ (see, e.g., ${ }^{1}$ ) and so $\mathcal{A}_{2}(\Phi) \Phi \in \mathcal{C}\left(\left[0, T^{*}\right] ; H_{\sigma}^{s-1} \times H_{\sigma}^{s-1}\right)$.

Finally, let us prove (3.9). Given $t \in\left[0, T^{*}\right]$, we multiply the equation in (3.10) by $\boldsymbol{\Phi}(t)=(\mathbf{u}(t), \mathbf{B}(t))$ in $H^{s} \times H^{s}$ :

$$
\frac{1}{2} \frac{d}{d t}\|\mathbf{\Phi}(t)\|_{s}^{2}+\left(\mathcal{A}_{1} \mathbf{\Phi}, \mathbf{\Phi}\right)_{s}=-\left(\mathcal{A}_{2}(\mathbf{\Phi}) \boldsymbol{\Phi}, \boldsymbol{\Phi}\right)_{s}(t)+(\mathbf{G}, \boldsymbol{\Phi})_{s}(t) .
$$

By using the estimate

$$
\left|((\mathbf{v} \cdot \nabla) \mathbf{w}, \mathbf{w})_{r}\right| \leq c\|\mathbf{v}\|_{r}\|\mathbf{w}\|_{r}^{2} \text { for all } \mathbf{v}, \mathbf{w} \in H_{\sigma}^{r} \text { with } r>\frac{5}{2},
$$

with $c>0$ a constant (proved in ${ }^{8}$ ) and since the operator $\mathcal{A}_{1}$ is dissipative, we obtain

$$
\frac{1}{2} \frac{d}{d t}\|\mathbf{\Phi}(t)\|_{s}^{2} \leq c\|\Phi\|_{s}^{3}+\|\mathbf{f}(t)\|_{s}\|\mathbf{\Phi}\|_{s}
$$


Using Young's inequality we find that $\|\boldsymbol{\Phi}(t)\|_{s}^{2}$ satisfies

$$
\frac{d}{d t}\|\mathbf{\Phi}(t)\|_{s} \leq c\|\boldsymbol{\Phi}\|_{s}+\|\mathbf{f}(t)\|_{s}, \quad\|\mathbf{\Phi}(0)\|_{s}=\left\|\left(\mathbf{u}_{0}, \mathbf{B}_{0}\right)\right\|_{s},
$$

and thus, $\|\boldsymbol{\Phi}(t)\|_{s} \leq \varphi(t)$, where $\varphi$ is the solution to the scalar Cauchy problem

$$
\frac{d \varphi}{d t}(t)=c^{\prime} \varphi^{2}(t)+\|\mathbf{f}(t)\|_{s}, \quad \varphi(0)=\left\|\left(\mathbf{u}_{0}, \mathbf{B}_{0}\right)\right\|_{s} .
$$

Since $\mathbf{f} \in L^{1}\left(0, T ; H_{\sigma}^{s}\right)$, it is clear that (3.16) admits an unique solution $\varphi$ defined in an interval $\left[0, T^{\prime}\right], T^{\prime}>0$ and that $T^{\prime}, \varphi$ are independent of the viscosities $\mathbf{R e}^{-1}, \mathrm{Rm}^{-1}$ and $\mu_{\alpha}$.

For the ideal case we have:

Theorem 3.7. Let be $T>0,\left(\mathbf{u}_{0}^{0}, \mathbf{B}_{0}^{0}\right) \in H^{s}\left(\mathbb{R}^{3}\right)^{3} \times H^{s}\left(\mathbb{R}^{3}\right)^{3}, s>$ $\frac{5}{2}$, such that $\nabla \cdot \mathbf{u}_{0}^{0}=\nabla \cdot \mathbf{B}_{0}^{0}=0$, and assume $\mathbf{f} \in \mathcal{C}\left([0, T] ; L^{2}\left(\mathbb{R}^{3}\right)\right)$ $\cap L^{1}\left(0, T ; H_{\sigma}^{s}\left(\mathbb{R}^{3}\right)\right)$. Then, there exists $T_{0} \in(0, T]$, just depending in the initial data, and an unique solution $\left(\mathbf{u}^{0}, \mathbf{B}^{0}\right)$ of (1.2) verifying

$$
\left(\mathbf{u}^{0}, \mathbf{B}^{0}\right) \in \mathcal{C}\left(\left[0, T_{0}\right] ; H^{s}\left(\mathbb{R}^{3}\right)^{3} \times H^{s}\left(\mathbb{R}^{3}\right)^{3}\right) \cap \mathcal{C}^{1}\left(\left[0, T_{0}\right] ;\left(H^{s-1}\left(\mathbb{R}^{3}\right)^{3}\right)^{2}\right)
$$

and such that, $\forall T^{*}<T_{0}$ the following estimate holds

$$
\int_{0}^{T^{*}}\left(\left\|\nabla \mathbf{u}^{0}(t)\right\|_{L^{\infty}}+\left\|\nabla \mathbf{B}^{0}(t)\right\|_{L^{\infty}}\right) d t<\infty .
$$

Moreover, if $T_{0}<\infty$, then at least one of the above integrals blows-up for some $T^{*} \leq T_{0}$.

Proof. As in the previous theorem, we consider the projected ideal MHD system into the divergence-free spaces. The resulting system can be regarded as the initial value problem

$$
\left\{\begin{array}{l}
\frac{d \Phi^{0}}{d t}+\mathcal{A}_{2}\left(\Phi^{0}\right) \Phi^{0}=\mathbf{G}(t) \\
\Phi^{0}(0)=\left(\mathbf{u}_{0}^{0}, \mathbf{B}_{0}^{0}\right)
\end{array}\right.
$$

in $H_{\sigma} \times H_{\sigma}$, with $\boldsymbol{\Phi}^{0}=\left(\mathbf{u}^{0}, \mathbf{B}^{0}\right)$. Again, we appeal to the existence and uniqueness results of Kato ${ }^{16}$ which apply in this case, provided that, for a fixed $\boldsymbol{\Phi} \in H_{\sigma}^{s} \times H_{\sigma}^{s}$, the operator $\left(-\mathcal{A}_{2}(\mathbf{\Phi}), D\left(\mathcal{A}_{2}(\mathbf{\Phi})\right)\right)$ generates a $\mathcal{C}^{0}$ semigroup on $H_{\sigma} \times H_{\sigma}$. We remark that this is the only point that remains 
to be proved for the $\mathcal{A}_{2}$ operator (Lemmas 3.2 and 3.4 hold if we replace $\mathcal{A}$ by $\left.\mathcal{A}_{2}\right)$. Due to the structure of $\mathcal{A}_{2}$, it suffices to show that, for a fixed $\mathbf{v} \in H_{\sigma}^{s}$, the operators $\left(P(\mathbf{v} \cdot \nabla), H_{\sigma}\right)$ and $\left(-P(\mathbf{v} \cdot \nabla), H_{\sigma}\right)$ are infinitesimal generators of $\mathcal{C}^{0}$-semigroups on $H_{\sigma}$.

Let $\mathbf{v} \in H_{\sigma}^{s}$ be fixed. It is a well-known result (see, e.g., ${ }^{10},{ }^{30}$ ) that the transport operator $(\mathbf{v} \cdot \nabla)$ with domain $H^{1} \hookrightarrow L^{2}\left(\mathbb{R}^{3}\right)^{3}$ generates a $\mathcal{C}^{0}$ isometries group on $L^{2}\left(\mathbb{R}^{3}\right)^{3}$ (note that the fixed vector $\mathbf{v}$ is solenoidal). Since $H_{\sigma}^{1}$ is a closed subset of $H^{1}$ and $P$ is unitary, the operator $P(\mathbf{v} \cdot \nabla)$ acting on $H_{\sigma}^{1}$ is also a generator of a $\mathcal{C}^{0}$-isometries group on $H_{\sigma}$. Furthermore, this result also holds for $\left(-P(\mathbf{v} \cdot \nabla), H_{\sigma}^{1}\right)$, which is possible since we are dealing with the generator of a group, and so we can conclude the desired result.

Then, by Kato ${ }^{16}$ (Theorems 6 and 7 ), there exists $T_{0} \in(0, T]$ and an unique $\boldsymbol{\Phi}^{0}=\left(\mathbf{u}^{0}, \mathbf{B}^{0}\right)$ solution of (3.18) such that

$$
\Phi^{0} \in \mathcal{C}\left(\left[0, T_{0}\right] ; H_{\sigma}^{s} \times H_{\sigma}^{s}\right) \cap \mathcal{C}^{1}\left(\left[0, T_{0}\right] ; H_{\sigma} \times H_{\sigma}\right) .
$$

Furthermore, we can use that $H^{s-1} \times H^{s-1}$ is a Banach algebra for $s>\frac{5}{2}$ and hence, by the above regularity, we get that

$$
\frac{d \Phi^{0}}{d t}=-\mathcal{A}_{2}(\Phi(t)) \Phi(t)+F(t) \in \mathcal{C}\left(\left[0, T_{0}\right] ; H_{\sigma}^{s-1} \times H_{\sigma}^{s-1}\right) .
$$

Finally, the Beale-Kato-Majda's type condition (3.17) for the ideal MHD equation is a result due to Caflisch, Klapper and Steele ${ }^{5}$.

\section{Convergence of the MHD Solutions to the Ideal MHD ones}

In this section we prove the convergence of the solutions $(\mathbf{u}, \mathbf{B})$ of the MHD for a viscous and resistive fluid found in Theorem 3.6, to the solutions $\left(\mathbf{u}_{0}, \mathbf{B}_{0}\right)$ of the ideal MHD equations (1.2). We shall start by establishing this convergence in the $L^{2}$-norm:

Theorem 4.1. Let $\left(\mathbf{u}_{0}, \mathbf{B}_{0}\right) \in H_{\sigma}^{s} \times H_{\sigma}^{s}, s>\frac{5}{2}$. Assume that $(\mathbf{u}, \mathbf{B})$ and $\left(\mathbf{u}^{0}, \mathbf{B}^{0}\right)$ are the solutions to (1.1) with Braginskii viscosity (2.4) and to (1.2) respectively, both with initial data $\left(\mathbf{u}_{0}, \mathbf{B}_{0}\right)$, given in Theorems 3.6 and 3.7. Then,

$$
\begin{aligned}
& \left\|\mathbf{u}(t)-\mathbf{u}^{0}(t)\right\|_{0}^{2}+S\left\|\mathbf{B}(t)-\mathbf{B}^{0}(t)\right\|_{0}^{2} \leq \\
& \quad \leq \exp \left(t+2 S^{\prime} \int_{0}^{t} \eta(s) d s\right) \int_{0}^{t}\left[k\left(\mu^{*}\right)^{2}\left\|\mathbf{u}^{0}\right\|_{2}^{2}+\frac{1}{\mathrm{Rm}^{2}}\left\|\nabla \mathbf{B}^{0}\right\|_{0}^{2}\right] d s,
\end{aligned}
$$

where $k$ is a positive constant, $\mu^{*}:=\max _{\alpha} \mu_{\alpha}, S^{\prime}=\max (1, S)$ and $\eta(t)=$ $\left\|\nabla \mathbf{u}^{0}(t)\right\|_{L^{\infty}}+\left\|\nabla \mathbf{B}^{0}(t)\right\|_{L^{\infty}}$. Furthermore, $\forall t \leq T^{\prime}$, with $T^{\prime}<+\infty$ satis- 
fying (3.17) we have

$$
(\mathbf{u}(t), \mathbf{B}(t)) \longrightarrow\left(\mathbf{u}^{0}(t), \mathbf{B}^{0}(t)\right) \text { in } L^{2}\left(\mathbb{R}^{3}\right)^{3} \times L^{2}\left(\mathbb{R}^{3}\right)^{3}
$$

when $R m \rightarrow+\infty$ and $\mu_{\alpha} \rightarrow 0, \alpha=0,1, \ldots, 4$, with convergence rates $o\left(\mu^{*}\right)+o\left(\frac{1}{\mathrm{Rm}}\right)$.

Proof. Let us denote by $(\mathbf{v}, \mathbf{C})$ the difference between the solutions of the MHD and ideal MHD systems with initial data $\left(\mathbf{u}_{0}, \mathbf{B}_{0}\right)$ stated in theorems 3.6 and 3.7, i.e., $(\mathbf{v}, \mathbf{C})=(\mathbf{u}, \mathbf{B})-\left(\mathbf{u}^{0}, \mathbf{B}^{0}\right)$; this couple satisfies

$$
\left\{\begin{aligned}
& \frac{\partial \mathbf{v}}{\partial t}+(\mathbf{v} \cdot \nabla) \mathbf{u}^{0}+(\mathbf{u} \cdot \nabla) \mathbf{v}-S(\mathbf{C} \cdot \nabla) \mathbf{B}^{0}-S(\mathbf{B} \cdot \nabla) \mathbf{C} \\
&+\frac{S}{2} \nabla\left(\mathbf{B}^{2}-\mathbf{B}^{0}\right)-\widehat{V} \mathbf{u}+\nabla\left(p-p^{0}\right)=0 \\
& \frac{\partial \mathbf{C}}{\partial t}+(\mathbf{v} \cdot \nabla) \mathbf{B}^{0}+(\mathbf{u} \cdot \nabla) \mathbf{C}-(\mathbf{C} \cdot \nabla) \mathbf{u}^{0}-(\mathbf{B} \cdot \nabla) \mathbf{v}-\frac{1}{\mathrm{Rm}} \Delta \mathbf{B}=0 \\
& \operatorname{div} \mathbf{v}=0, \operatorname{div} \mathbf{C}=0 \\
& \mathbf{v}_{/ t=0}=\mathbf{C}_{/ t=0}=\mathbf{0}
\end{aligned}\right.
$$

Let us fix $t \in\left(0, T^{\prime}\right]$ and multiply in $L^{2}\left(\mathbb{R}^{3}\right)^{3}$ the first two equations in (4.20) by $\mathbf{v}(t)$ and $\mathbf{B}(t)$ respectively. Then

$$
\begin{aligned}
\frac{1}{2} \frac{d}{d t}\|\mathbf{v}(t)\|_{0}^{2} & =(\widehat{V} \mathbf{u}(t), \mathbf{v}(t))-\left((\mathbf{v} \cdot \nabla) \mathbf{u}^{0}, \mathbf{v}\right)(t) \\
& +S\left((\mathbf{C} \cdot \nabla) \mathbf{B}^{0}, \mathbf{v}\right)(t)+S((\mathbf{B} \cdot \nabla) \mathbf{C}, \mathbf{v})(t)
\end{aligned}
$$

and

$$
\begin{aligned}
\frac{1}{2} \frac{d}{d t}\|\mathbf{C}(t)\|_{0}^{2} & =\frac{1}{\mathrm{Rm}}(\Delta \mathbf{B}(t), \mathbf{C}(t))-\left((\mathbf{v} \cdot \nabla) \mathbf{B}^{0}, \mathbf{C}\right)(t) \\
& +\left((\mathbf{C} \cdot \nabla) \mathbf{u}^{0}, \mathbf{C}\right)(t)+((\mathbf{B} \cdot \nabla) \mathbf{v}, \mathbf{C})(t)
\end{aligned}
$$

where we have used that the terms $\left(\nabla\left(p-p^{0}\right), \mathbf{v}(t)\right),((\mathbf{u}(t) \cdot \nabla) \mathbf{v}(t), \mathbf{v}(t))$, and $-\frac{S}{2}\left(\nabla\left(\mathbf{B}^{2}-\left(\mathbf{B}^{0}\right)^{2}\right)(t), \mathbf{v}(t)\right)$ vanish since $\mathbf{v}(t)$ and $\mathbf{u}(t)$ are divergencefree (this can be easily seen by integrating by parts). Similarly, $-((\mathbf{u} \cdot \nabla) \mathbf{C}, \mathbf{C}(t))$ vanish too.

We shall estimate the integrals in (4.21) and (4.22), in order to derive an energy inequality for $\mathbf{v}(t)$ and $\mathbf{C}(t)$ in $L^{2}\left(\mathbb{R}^{3}\right)^{3}$. Let us start with the terms containing the viscosity operators; we know (Lemma 2.1) that these 
operators are dissipative in $L^{2}$ and continuous from $H^{2}$ to $L^{2}$, hence we can appeal to Hölder's and Young's inequalities to get

$$
\begin{aligned}
(\widehat{V} \mathbf{u}(t), \mathbf{v}(t)) & =(\widehat{V} \mathbf{v}(t), \mathbf{v}(t))+\left(\widehat{V} \mathbf{u}^{0}(t), \mathbf{v}(t)\right) \\
& \leq-\left(\min _{\alpha} \mu_{\alpha}\right)\|\nabla \mathbf{v}(t)\|_{0}^{2}+k\left(\max _{\alpha} \mu_{\alpha}\right)\left\|\mathbf{u}^{0}(t)\right\|_{2}\|\mathbf{v}(\mathbf{t})\|_{0} \\
& \leq-\left(\min _{\alpha} \mu_{\alpha}\right)\|\nabla \mathbf{v}(t)\|_{0}^{2}+\frac{k}{2}\left(\max _{\alpha} \mu_{\alpha}\right)^{2}\left\|\mathbf{u}^{0}(t)\right\|_{2}^{2}+ \\
& +\frac{1}{2}\|\mathbf{v}(t)\|_{0}^{2}
\end{aligned}
$$

in the case of the fluid viscosity (here, $k$ is the continuity constant in Lemma 2.1). Similarly, for the magnetic viscosity term we have

$$
\frac{1}{\mathrm{Rm}}(\Delta \mathbf{B}(t), \mathbf{C}(t)) \leq-\frac{1}{\mathrm{Rm}}\|\nabla \mathbf{C}(t)\|_{0}^{2}+\frac{1}{2 \mathrm{Rm}^{2}}\left\|\Delta \mathbf{B}^{0}(t)\right\|_{0}^{2}+\frac{1}{2}\|\mathbf{C}(t)\|_{0}^{2} .
$$

We proceed now to estimate the transport terms in (4.21) and (4.22). For this purpose, we apply again Cauchy-Schwarz's and Young's inequalities and we arrive to

(i) $\left|\left((\mathbf{v} \cdot \nabla) \mathbf{u}^{0}, \mathbf{v}\right)\right| \leq\left\|\nabla \mathbf{u}^{0}(t)\right\|_{\infty}\|\mathbf{v}(t)\|_{0}^{2}$,

(ii) $S\left|\left((\mathbf{C} \cdot \nabla) \mathbf{B}^{0}, \mathbf{v}\right)\right| \leq S\left\|\nabla \mathbf{B}^{0}(t)\right\|_{\infty}\|\mathbf{v}(t)\|_{0}\|\mathbf{C}(t)\|_{0}$

$$
\leq \frac{S}{2}\left\|\nabla \mathbf{B}^{0}(t)\right\|_{\infty}\left(\|\mathbf{v}(t)\|_{0}^{2}+\|\mathbf{C}(t)\|_{0}^{2}\right)
$$

(iii) $\left|\left((\mathbf{v} \cdot \nabla) \mathbf{B}^{0}, \mathbf{C}\right)(t)\right| \leq\left\|\nabla \mathbf{B}^{0}(t)\right\|_{\infty}\|\mathbf{v}(t)\|_{0}\|\mathbf{C}(t)\|_{0}$

$$
\leq \frac{1}{2}\left\|\nabla \mathbf{B}^{0}(t)\right\|_{\infty}\left(\|\mathbf{v}(t)\|_{0}^{2}+\|\mathbf{C}(t)\|_{0}^{2}\right),
$$

(iv) $\left((\mathbf{C} \cdot \nabla) \mathbf{u}^{0}, \mathbf{C}\right)(t) \leq\left\|\nabla \mathbf{u}^{0}(t)\right\|_{\infty}\|\mathbf{C}(t)\|$.

Putting together the preceding estimates and (4.23), (4.24) we obtain:

$$
\begin{aligned}
\frac{1}{2} \frac{d}{d t} & \left(\|\mathbf{v}(t)\|_{0}^{2}+S\|\mathbf{C}(t)\|_{0}^{2}\right)+\left(\min _{\alpha} \mu_{\alpha}\right)\|\nabla \mathbf{v}\|_{0}^{2}+\frac{S}{\operatorname{Rm}}\|\nabla \mathbf{C}(t)\|_{0}^{2} \leq \\
& \leq \frac{1}{2}\left(k\left(\max _{\alpha} \mu_{\alpha}\right)^{2}\left\|\mathbf{u}^{0}\right\|_{2}^{2}+\frac{S}{\operatorname{Rm}^{2}}\left\|\Delta \mathbf{B}^{0}\right\|_{0}^{2}\right)+\frac{1}{2}\left(\|\mathbf{v}(t)\|_{0}^{2}+S\|\mathbf{C}\|_{0}^{2}\right) \\
& +\left\|\nabla \mathbf{u}^{0}(t)\right\|_{\infty}\left(\|\mathbf{v}(t)\|_{0}^{2}+S\|\mathbf{C}\|_{0}^{2}\right)+S\left\|\nabla \mathbf{B}^{0}(t)\right\|_{\infty}\left(\|\mathbf{v}(t)\|_{0}^{2}+\|\mathbf{C}(t)\|_{0}^{2}\right) \\
& +S((\mathbf{B} \cdot \nabla) \mathbf{C}, \mathbf{v}(t))+S((\mathbf{B} \cdot \nabla) \mathbf{v}, \mathbf{C}(t))
\end{aligned}
$$

(We have used here that $S$ is a positive constant). 
Let us set $S^{\prime}=\max (1, S)$. By dropping the positive terms in the left hand side of (4.26) and using the identity $((\mathbf{B} \cdot \nabla) \mathbf{C}, \mathbf{v}(t))=-((\mathbf{B} \cdot \nabla) \mathbf{v}, \mathbf{C}(t))$ (see, e.g. , Temam ${ }^{29}$ ), we can write

$$
\begin{gathered}
\frac{d}{d t}\left(\|\mathbf{v}(t)\|_{0}^{2}+S\|\mathbf{C}(t)\|_{0}^{2}\right) \leq\left(k\left(\max _{\alpha} \mu_{\alpha}\right)^{2}\left\|\mathbf{u}^{0}(t)\right\|_{2}^{2}+\frac{S}{\mathrm{Rm}^{2}}\left\|\Delta \mathbf{B}^{0}(t)\right\|_{0}^{2}\right)+ \\
+\widehat{\eta}(t)\left(\|\mathbf{v}(t)\|_{0}^{2}+S\|\mathbf{C}(t)\|_{0}^{2}\right)
\end{gathered}
$$

where $\widehat{\eta}(t):=1+2\left\|\nabla \mathbf{u}^{0}(t)\right\|_{\infty}+2 S^{\prime}\left\|\nabla \mathbf{B}^{0}(t)\right\|_{\infty}$. We note that $\eta$ is an integrable function on $[0, t]$ for $t \leq T^{\prime}$ satisfying (3.17). Thus, we can apply to Gronwall's inequality to

$$
\frac{d}{d t} \varphi(t) \leq\left(k\left(\max _{\alpha} \mu_{\alpha}\right)^{2}\left\|\mathbf{u}^{0}(t)\right\|_{2}^{2}+\frac{S}{\mathrm{Rm}^{2}}\left\|\Delta \mathbf{B}^{0}(t)\right\|_{0}^{2}\right)+\widehat{\eta}(t) \varphi(t)
$$

where $\varphi(t):=\|\mathbf{v}(t)\|_{0}^{2}+S\|\mathbf{C}(t)\|_{0}^{2}$ and hence we obtain (4.19). The convergence in the statement of the theorem follows from this inequality.

When the viscosity in (1.1) is given by (2.3) we arrive to an analogous result:

Theorem 4.2. Let be $\left(\mathbf{u}_{0}, \mathbf{B}_{0}\right) \in H_{\sigma}^{s} \times H_{\sigma}^{s}, s>\frac{5}{2}$. Assume that $\mathbf{F}=\mathbf{R e}^{-1} \Delta$ in (1.1) and that $(\mathbf{u}, \mathbf{B})$ and $\left(\mathbf{u}^{0}, \mathbf{B}^{0}\right)$ are the solutions to the MHD and ideal MHD systems respectively, with initial data $\left(\mathbf{u}_{0}, \mathbf{B}_{0}\right)$, given by Theorems 3.6 and 3.7. Then,

$$
\begin{aligned}
& \left\|\left(\mathbf{u}-\mathbf{u}^{0}\right)(t)\right\|_{0}^{2}+S\left\|\left(\mathbf{B}-\mathbf{B}^{0}\right)(t)\right\|_{0}^{2} \leq \\
& \quad \leq \exp \left(t+2 S^{\prime} \int_{0}^{t} \eta(s) d s\right) \int_{0}^{t}\left[\frac{1}{\mathbf{R e}^{2}}\left\|\nabla \mathbf{u}^{0}\right\|_{0}^{2}+\frac{1}{\mathrm{Rm}^{2}}\left\|\nabla \mathbf{B}^{0}\right\|_{0}^{2}\right] d s,
\end{aligned}
$$

where $S^{\prime}=\max (1, S)$ and $\eta(t)=\left\|\nabla \mathbf{u}^{0}(t)\right\|_{L^{\infty}}+\left\|\nabla \mathbf{B}^{0}(t)\right\|_{L^{\infty}}$. In particular, $\forall t \leq T^{\prime}$ with $T^{\prime}<+\infty$ verifying (3.17) we conclude that

$$
(\mathbf{u}(t), \mathbf{B}(t)) \longrightarrow\left(\mathbf{u}^{0}(t), \mathbf{B}^{0}(t)\right) \text { in } L^{2}\left(\mathbb{R}^{3}\right)^{3} \times L^{2}\left(\mathbb{R}^{3}\right)^{3}
$$

as $\mathbf{R e}, R m \rightarrow+\infty$ with a convergence rate $o\left(\frac{1}{\mathbf{R e}}\right)+o\left(\frac{1}{\mathrm{Rm}}\right)$.

Proof. Let be $(\mathbf{v}, \mathbf{C})=(\mathbf{u}, \mathbf{B})-\left(\mathbf{u}_{0}, \mathbf{B}_{0}\right)$ as before. The proof of this theorem is analogous to the previous one, being the dissipative term in (4.21) the only difference. To estimate this term we proceed as in (4.24), i.e.,

$$
\begin{array}{r}
\frac{1}{\operatorname{Re}}(\Delta \mathbf{u}(t), \mathbf{v}(t)) \leq-\frac{1}{\operatorname{Re}}\|\nabla \mathbf{v}(t)\|_{0}^{2}+\frac{1}{\operatorname{Re}}\left(\Delta \mathbf{u}^{0}, \mathbf{v}\right) \leq \\
-\frac{1}{\mathbf{R e}}\|\nabla \mathbf{v}(t)\|_{0}^{2}+\frac{1}{2 \mathbf{R e}}\left\|\Delta \mathbf{u}^{0}\right\|_{0}^{2}+\frac{1}{2}\|\mathbf{v}(t)\|_{0}^{2},
\end{array}
$$


and hence, repeating the calculus as in theorem 4.2 we obtain the following energy estimate:

$$
\begin{aligned}
\frac{d}{d t}\left(\|\mathbf{v}(t)\|_{0}^{2}+S\|\mathbf{C}(t)\|_{0}^{2}\right) & \leq\left(\frac{1}{\mathbf{R e}}\left\|\Delta \mathbf{u}^{0}\right\|_{0}^{2}+\frac{S}{\operatorname{Rm}^{2}}\left\|\Delta \mathbf{B}^{0}\right\|_{0}^{2}\right)+ \\
& +\widehat{\eta}(t)\left(\|\mathbf{v}(t)\|_{0}^{2}+S\|\mathbf{C}(t)\|_{0}^{2}\right),
\end{aligned}
$$

with $\widehat{\eta}$ as in the preceding proof. Then, we can apply Gronwall's inequality to $\|\mathbf{v}(t)\|_{0}^{2}+S\|\mathbf{C}(t)\|_{0}^{2}$ which yields to $(4.27)$.

Finally, we study the convergence in the $H^{s}$ norm. We have:

Theorem 4.3. Let us assume $\left(\mathbf{u}_{0}, \mathbf{B}_{0}\right) \in H_{\sigma}^{s} \times H_{\sigma}^{s}$ with $s>\frac{9}{2}$. Then, if $\left(\mathbf{u}^{0}, \mathbf{B}^{0}\right)$ and $(\mathbf{u}, \mathbf{B})$ are as in theorem 4.2 , the following inequality holds

$$
\begin{aligned}
& \left\|\left(\mathbf{u}-\mathbf{u}^{0}\right)(t)\right\|_{s-2}^{2}+S\left\|\left(\mathbf{B}-\mathbf{B}^{0}\right)(t)\right\|_{s-2}^{2} \leq \\
& \quad \leq \exp \left(2 \int_{0}^{t} \xi(\tau) d \tau\right) \int_{0}^{t}\left[k\left(\mu^{*}\right)^{2}\left\|\mathbf{u}^{0}\right\|_{s}^{2}+\frac{S}{\mathrm{Rm}^{2}}\left\|\mathbf{B}^{0}\right\|_{s}^{2}\right] d \tau
\end{aligned}
$$

where $k$ is a positive constant, $\mu^{*}:=\max _{\alpha} \mu_{\alpha}$ and $\xi \in \mathcal{C}\left(\left[0, T^{\prime}\right]\right)$ does not depend on $\mathrm{Rm}$ or $\mu_{\alpha}$. Moreover, $\forall t \leq T^{\prime}$ with $T^{\prime}<+\infty$ satisfying (3.17) we have

$$
(\mathbf{u}(t), \mathbf{B}(t)) \longrightarrow\left(\mathbf{u}^{0}(t), \mathbf{B}^{0}(t)\right) \text { in } H^{s-2}\left(\mathbb{R}^{3}\right)^{3} \times H^{s-2}\left(\mathbb{R}^{3}\right)^{3} \text {-strong }
$$

as $\mathrm{Rm}, \mathbf{R e} \rightarrow+\infty$ with a convergence rate of $o\left(\frac{1}{\mathbf{R e}}\right)+o\left(\frac{1}{\mathrm{Rm}}\right)$.

Proof. As in the previous cases, we consider the couple $(\mathbf{v}, \mathbf{C}):=(\mathbf{u}, \mathbf{B})-$ $\left(\mathbf{u}^{0}, \mathbf{B}^{0}\right)$ which satisfies the system (4.20). Multiplying (4.20) by $\mathbf{v}(t)$ and $\mathbf{C}(t)$ with the inner product of $H^{s-2}$, for $t \in\left(0, T^{\prime}\right]$ fixed, we obtain

$$
\begin{array}{r}
\frac{1}{2} \frac{d}{d t}\|\mathbf{v}(t)\|_{s-2}^{2}=(\widehat{V} \mathbf{u}, \mathbf{v})_{s-2}+S\left((\mathbf{C} \cdot \nabla) \mathbf{B}^{0}, \mathbf{v}\right)_{s-2}+S((\mathbf{B} \cdot \nabla) \mathbf{C}, \mathbf{v})_{s-2} \\
-\left((\mathbf{v} \cdot \nabla) \mathbf{u}^{0}, \mathbf{v}\right)_{s-2}-((\mathbf{u} \cdot \nabla) \mathbf{v}, \mathbf{v})_{s-2}
\end{array}
$$

and

$$
\begin{gathered}
\frac{1}{2} \frac{d}{d t}\|\mathbf{C}(t)\|_{s-2}^{2}=\frac{1}{\mathrm{Rm}}(\Delta \mathbf{B}, \mathbf{C})_{s-2}+\left((\mathbf{C} \cdot \nabla) \mathbf{u}^{0}, \mathbf{C}\right)_{s-2}+((\mathbf{B} \cdot \nabla) \mathbf{v}, \mathbf{C})_{s-2} \\
-\left((\mathbf{v} \cdot \nabla) \mathbf{B}^{0}, \mathbf{C}\right)_{s-2}-((\mathbf{u} \cdot \nabla) \mathbf{C}, \mathbf{C})_{s-2} .
\end{gathered}
$$

As before, we have used that $\nabla \cdot \mathbf{v}(t)=\nabla \cdot \mathbf{C}(t)=0$ and so the terms $\left(\nabla\left(p-p^{0}\right), \mathbf{v}(t)\right)_{s-2}$ and $-\frac{S}{2}\left(\nabla\left(\mathbf{B}^{2}-\left(\mathbf{B}^{0}\right)^{2}\right)(t), \mathbf{v}(t)\right)_{s-2}$ vanish. Nevertheless, this is no longer true for the products $((\mathbf{u}(t) \cdot \nabla) \mathbf{v}(t), \mathbf{v}(t))_{s-2}$ and $((\mathbf{u} \cdot \nabla) \mathbf{C}, \mathbf{C}(t))_{s-2}$. 
The viscosity operators $-\widehat{V}$ and $-\Delta$ are dissipative in the space $H^{s-2}$. This is well-known for the Laplacian, while for the Braginskii's operator it comes from the fact that $-\widehat{V}$ being dissipative in $L^{2}\left(\mathbb{R}^{3}\right)^{3}$ and commuting with $\mathrm{S}^{s-2}=(1-\Delta)^{\frac{s-2}{2}}$. Thus,

$$
\begin{aligned}
(\widehat{V} \mathbf{u}, \mathbf{v})_{s-2}(t) & =(\widehat{V} \mathbf{v}(t), \mathbf{v}(t))_{s-2}+\left(\widehat{V} \mathbf{u}^{0}(t), \mathbf{v}(t)\right)_{s-2} \leq \\
& \leq\left\|\widehat{V} \mathbf{u}^{0}(t)\right\|_{s-2}\|\mathbf{v}(t)\|_{s-2} \\
& \leq k^{\prime \prime}\left(\max _{\alpha=0, \ldots, 4} \mu_{\alpha}\right)\left\|\mathbf{u}^{0}(t)\right\|_{s}\|\mathbf{v}(t)\|_{s-2} \\
& \leq \frac{k^{\prime \prime}}{2}\left(\max _{\alpha=0, \ldots, 4} \mu_{\alpha}\right)^{2}\left\|\mathbf{u}^{0}(t)\right\|_{s}^{2}+\frac{1}{2}\|\mathbf{v}(t)\|_{s-2}^{2}
\end{aligned}
$$

where we have also used that $\widehat{V}$ is a continuous operator from $H_{\sigma}^{s}$ into $H_{\sigma}^{s-2}$ being $k^{\prime \prime}$ the continuity constant (see Lemma 2.1). Also,

$$
\begin{aligned}
\frac{1}{\mathrm{Rm}}(\Delta \mathbf{B}(t), \mathbf{C}(t))_{s-2} & =\frac{1}{\mathrm{Rm}}(\Delta \mathbf{C}(t), \mathbf{C}(t))_{s-2}+\frac{1}{\mathrm{Rm}}\left(\Delta \mathbf{B}^{0}(t), \mathbf{C}(t)\right)_{s-2} \leq \\
& \leq \frac{-1}{\mathrm{Rm}}\|\nabla \mathbf{C}(t)\|_{s-2}^{2}+\frac{1}{2 \mathrm{Rm}^{2}}\left\|\mathbf{B}^{0}(t)\right\|_{s}^{2}+\frac{1}{2}\|\mathbf{C}(t)\|_{s-2}^{2} .
\end{aligned}
$$

From the choice of $s$ it follows that $H^{s-2}$ is a Banach algebra (note that $\left.s-2>\frac{3}{2}\right)$, hence

$$
\begin{aligned}
\left|\left((\mathbf{v} \cdot \nabla) \mathbf{u}^{0}, \mathbf{v}\right)_{s-2}(t)\right| & \leq\left\|\nabla \mathbf{u}^{0}(t)\right\|_{s-2}\|\mathbf{v}(t)\|_{s-2}^{2}, \\
\left|\left((\mathbf{C} \cdot \nabla) \mathbf{u}^{0}, \mathbf{C}\right)_{s-2}(t)\right| & \leq\left\|\nabla \mathbf{u}^{0}(t)\right\|_{s-2}\|\mathbf{C}(t)\|_{s-2}^{2} .
\end{aligned}
$$

Analogously, Young's inequality yields to

$$
\begin{aligned}
\left|\left((\mathbf{C} \cdot \nabla) \mathbf{B}^{0}, \mathbf{v}\right)_{s-2}(t)\right| & \leq\left\|\nabla \mathbf{B}^{0}(t)\right\|_{s-2}\|\mathbf{C}(t)\|_{s-2}\|\mathbf{v}(t)\|_{s-2} \\
\leq & \frac{1}{2}\left\|\nabla \mathbf{B}^{0}(t)\right\|_{s-2}\left(\|\mathbf{C}(t)\|_{s-2}^{2}+\|\mathbf{v}(t)\|_{s-2}^{2}\right), \\
\left|\left((\mathbf{C} \cdot \nabla) \mathbf{B}^{0}, \mathbf{v}\right)_{s-2}(t)\right| & \leq \frac{1}{2}\left\|\nabla \mathbf{B}^{0}(t)\right\|_{s-2}\left(\|\mathbf{C}(t)\|_{s-2}^{2}+\|\mathbf{v}(t)\|_{s-2}^{2}\right) .
\end{aligned}
$$

For the remaining terms in (4.30) and (4.31) we use the estimate (3.14) to get

$$
\begin{aligned}
\left|((\mathbf{u} \cdot \nabla) \mathbf{v}, \mathbf{v})_{s-2}(t)\right| & \leq c\|\mathbf{u}(t)\|_{s-2}\|\mathbf{v}(t)\|_{s-2}^{2}, \\
\left|((\mathbf{u} \cdot \nabla) \mathbf{C}, \mathbf{C})_{s-2}(t)\right| & \leq c\|\mathbf{u}(t)\|_{s-2}\|\mathbf{C}(t)\|_{s-2}^{2}
\end{aligned}
$$


and also,

$$
\begin{gathered}
((\mathbf{B} \cdot \nabla) \mathbf{C}, \mathbf{v})_{s-2}(t)+((\mathbf{B} \cdot \nabla) \mathbf{v}, \mathbf{C})_{s-2}(t) \leq((\mathbf{B} \cdot \nabla)(\mathbf{v}+\mathbf{C}), \mathbf{v}+\mathbf{C})_{s-2}(t) \\
-((\mathbf{B} \cdot \nabla) \mathbf{v}, \mathbf{v})_{s-2}(t)-((\mathbf{B} \cdot \nabla) \mathbf{C}, \mathbf{C})_{s-2}(t) \\
\leq 3 c\|\mathbf{B}(t)\|_{s-2}\left(\|\mathbf{v}(t)\|_{s-2}^{2}+\|\mathbf{C}(t)\|_{s-2}^{2}\right)
\end{gathered}
$$

where $c$ is the constant in (3.14).

Collecting all the estimates that we have obtained for the terms appearing in (4.30) and (4.31) we arrive to

$$
\begin{aligned}
\frac{1}{2} \frac{d}{d t}\left(S\|\mathbf{C}(t)\|_{s-2}^{2}+\|\mathbf{v}\|_{s-2}^{2}\right) \leq \frac{1}{2} k^{\prime \prime}\left(\max _{\alpha=0, \ldots, 4} \mu_{\alpha}\right)^{2}\left\|\mathbf{u}^{0}(t)\right\|_{s}^{2}+\frac{S}{2 \mathrm{Rm}^{2}}\left\|\mathbf{B}^{0}(t)\right\|_{s}^{2} \\
+S c\left(\|\mathbf{v}(t)\|_{s-2}^{2}+\|\mathbf{C}(t)\|_{s-2}^{2}\right)\left(\|\mathbf{u}(t)\|_{s-2}+3\|\mathbf{B}(t)\|_{s-2}\right) \\
+\left(\left\|\nabla \mathbf{u}^{0}(t)\right\|_{s-2}+S^{\prime}\left\|\nabla \mathbf{B}^{0}(t)\right\|_{s-2}\right)\left(\|\mathbf{v}(t)\|_{s-2}^{2}+\|\mathbf{C}(t)\|_{s-2}^{2}\right) \\
+\frac{1}{2}\left(\|\mathbf{v}(t)\|_{s-2}^{2}+S\|\mathbf{C}(t)\|_{s-2}^{2}\right) \\
\leq \frac{1}{2} k\left(\mu^{*}\right)^{2}\left\|\mathbf{u}^{0}(t)\right\|_{s}^{2}+\frac{S}{2 \mathrm{Rm}^{2}}\left\|\mathbf{B}^{0}(t)\right\|_{s}^{2}+\left(\|\mathbf{v}\|_{m-2}^{2}+S\|\mathbf{C}\|_{m-2}^{2}\right) \xi(t)
\end{aligned}
$$

where $\xi(t):=K\left(\|\mathbf{u}(t)\|_{s-2}+\|\mathbf{B}(t)\|_{s-2}+\left\|\nabla \mathbf{u}^{0}(t)\right\|_{s-2}+\left\|\nabla \mathbf{B}^{0}(t)\right\|_{s-2}+\frac{1}{2}\right)$ and $K:=\max (1, S, 3 c)$ is a positive constant that just depend on $S$ and on the exponent $s$. Moreover, from the regularity of $(\mathbf{u}, \mathbf{B})$ and $\left(\mathbf{u}^{0}, \mathbf{B}^{0}\right)$, it follows that $\xi:\left(0, T^{\prime}\right) \longrightarrow \mathbb{R}$ is continuous and uniformly bounded with respect to $\mu_{\alpha}$ and $\mathrm{Rm}, \alpha=0, \ldots, 4$, in $\left[0, T^{\prime}\right]$ (see Theorem 3.6, (3.9)).

Thus, we can apply Gronwall's inequality to $\|\mathbf{v}\|_{s-2}^{2}+S\|\mathbf{C}(t)\|_{s-2}^{2}$ and using that $(\mathbf{v}(0), \mathbf{C}(0))=(\mathbf{0}, \mathbf{0})$, we get

$$
\begin{gathered}
\|\mathbf{v}(t)\|_{s-2}^{2}+S\|\mathbf{C}(t)\|_{s-2}^{2} \leq \\
\leq \exp \left(2 \int_{0}^{t} \xi(\tau) d \tau\right) \int_{0}^{t}\left[k\left(\max _{\alpha=0, \ldots, 4} \mu_{\alpha}\right)^{2}\left\|\mathbf{u}^{0}\right\|_{s}^{2}+\frac{S}{\mathrm{Rm}^{2}}\left\|\mathbf{B}^{0}\right\|_{s}^{2}\right] d \tau,
\end{gathered}
$$

which concludes the proof of the theorem.

Arguing in a similar way, for $\mathbf{F}=\frac{1}{\mathbf{R e}} \Delta$, we obtain

Theorem 4.4. Assume $\left(\mathbf{u}_{0}, \mathbf{B}_{0}\right) \in H_{\sigma}^{s} \times H_{\sigma}^{s}$ with $s>\frac{9}{2}$, and let be $\left(\mathbf{u}^{0}, \mathbf{B}^{0}\right)$ and $(\mathbf{u}, \mathbf{B})$ as in Theorem 4.2. Then

$$
\begin{aligned}
& \left\|\left(\mathbf{u}-\mathbf{u}^{0}\right)(t)\right\|_{s-2}^{2}+S\left\|\left(\mathbf{B}-\mathbf{B}^{0}\right)(t)\right\|_{s-2}^{2} \leq \\
& \quad \leq \exp \left(2 \int_{0}^{t} \xi^{\prime \prime}(\tau) d \tau\right) \int_{0}^{t}\left[\frac{1}{\mathbf{R e}^{2}}\left\|\nabla \mathbf{u}^{0}\right\|_{s-2}^{2}+\frac{S}{\mathrm{Rm}^{2}}\left\|\nabla \mathbf{B}^{0}\right\|_{s-2}^{2}\right] d \tau
\end{aligned}
$$


where $\xi^{\prime \prime} \in \mathcal{C}\left(\left[0, T^{\prime}\right]\right)$ does not depend on Rm or Re. Moreover, $\forall t \leq T^{\prime}$ with $T^{\prime}<+\infty$ verifying (3.17), (4.32) yields to

$$
(\mathbf{u}(t), \mathbf{B}(t)) \longrightarrow\left(\mathbf{u}^{0}(t), \mathbf{B}^{0}(t)\right) \text { in } H^{s-2}\left(\mathbb{R}^{3}\right)^{3} \times H^{s-2}\left(\mathbb{R}^{3}\right)^{3}
$$

as $\mathbf{R e}, R m \rightarrow+\infty$ with a convergence rate $o\left(\frac{1}{\mathbf{R e}}\right)+o\left(\frac{1}{\mathrm{Rm}}\right)$.

Remark 4.5. In order to simplify the exposition, we have considered the same initial data for both, the MHD and the ideal MHD systems. Nevertheless, the convergences and convergence rates obtained in theorems 4.1 (respectively, 4.2) and 4.3 (respect., 4.4) remain true if we consider different initial data $\left(\mathbf{u}_{0}^{\text {vis }}, \mathbf{B}_{0}^{\text {vis }}\right)$ and $\left(\mathbf{u}_{0}^{0}, \mathbf{B}_{0}^{0}\right)$ for (1.1) and (1.2) respectively, verifying

$$
\left\|\mathbf{u}_{0}^{v i s}-\mathbf{u}_{0}^{0}\right\|_{0}+\left\|\mathbf{B}_{0}^{v i s}-\mathbf{B}_{0}^{0}\right\|_{0} \rightarrow 0 \text { as } o\left(\mu^{*}\right)+o\left(\frac{1}{\mathrm{Rm}}\right)
$$

when $\mathrm{Rm} \rightarrow \infty$ and $\mu_{\alpha} \rightarrow 0, \alpha=0,1, \ldots, 4$ (respect., as $o\left(\frac{1}{\mathbf{R e}}\right)+o\left(\frac{1}{\mathrm{Rm}}\right)$ when $\operatorname{Re}, \operatorname{Rm} \rightarrow+\infty)$, for the first case, or

$$
\left\|\mathbf{u}_{0}^{v i s}-\mathbf{u}_{0}^{0}\right\|_{s-2}+\left\|\mathbf{B}_{0}^{v i s}-\mathbf{B}_{0}^{0}\right\|_{s-2} \rightarrow 0 \text { as } o\left(\mu^{*}\right)+o\left(\frac{1}{\mathrm{Rm}}\right)
$$

in the second case.

Remark 4.6. The same remark holds if we consider different force terms $\mathbf{f}$ for the MHD system and for the ideal MHD one.

\section{Acknowledgment}

The research of the authors is partially supported by Project FTN2000-2043 of the SGPI of Spain. The second author has been also partially supported by a Predoctoral Fellowship of the Universidad Complutense de Madrid (19972000).

\section{References}

1. R. Adams, Sobolev Spaces (Acad. Press, 1975).

2. D. Biskamp, Nonlinear Magnetohydrodynamics (Cambridge Univ. Press, 1997).

3. S.I. Braginskii, in Transport processes in a plasma Vol. 1, Reviews of a Plasma Physics (Consultants Bureau NY, 1965) pp. 205-311.

4. H. Brézis, Analyse Fonctionnelle (Masson Editeur, 1983).

5. R.E. Caflisch, I. Klapper and G. Steele, Remarks on Singularities, Dimension and Energy Dissipation for Ideal Hydrodynamics and MHD, Commun. Math. Phys., 184 (1997) 443-455.

6. M. Cannone, Ondelettes, Paraproduits et Navier-Stokes, Nouveaux Essais (Diderot Ed., Arts et Sciences, 1995). 
7. T. Cazenave and A. Haraux, An Introduction to Semilinear Evolution Equations (Clarendon Press, Oxford, 1998).

8. P.Constantin and C.Foias, Navier-Stokes Equations (The University of Chicago Press, 1988)

9. T.G. Cowling, Magnetohidrodynamics (John Wiley \& Sons Inc. NY, 1965).

10. R. Dautray and J.L. Lions, Analyse Mathématique et Calcul Numérique pour les Sciences et les Techniques 9 (Ed. Masson, 1988).

11. J.I. Diaz, Simulación numérica de un Equilibrio de un Plasma Magnéticamente Confinado en un Stellarator, Informe \#2, CIEMAT (1998).

12. G. Duvaut and J.L. Lions, Inéquations en thermoélasticité et magnétohydrodynamique, Arc. Rational Mech. Anal. 46 (1972) 241-279.

13. J.P. Freidberg, Ideal Magnetohydrodinamic Theory of Magnetic Fusion Systems Rev. Moderm Physics Vol. 54, No. 3 (The American Physical Society, 1982).

14. D. Gilbarg and N. Trudinger, Elliptic Partial Differential Equations of Second Order (Springer-Verlag, 1983)

15. R.D. Hazeltine and J.D. Meiss, Plasma Confinement (Addison-Wesley Publishing Co., 1992).

16. T. Kato, Quasi-linear equations of evolution, with applications to partial differential equations, Lect. Notes in Math. Vol. 448 (Springer-Verlag, 1975) pp. 25-70.

17. T. Kato, Nonstationary Flows of Viscous and Ideal Fluids in $R^{3}$, J. Functional Analysis 9 (1972) 296-305.

18. T. Kato, Perturbation Theory for Linear Operators(Springer, 1966).

19. T. Kato and G. Ponce, Commutator estimates and the Euler and Navier-Stokes equations, Comm. Pure and Appl. Math. XLI (1988) 891-907.

20. J. Rauch, Partial Differential Equations, ( Springer-Verlag NY, 1997).

21. P.Schmidt, On a Magnetohydrodynamic problem of Euler type,J. Diff. Eq. $\mathbf{7 4}$ (1988) 318-335.

22. P. Secchi, On the Equations of Ideal Incompressible Magneto-Hydrodynamics, Rend. Sem. Ma. Univ. Padova 90 (1993) 104-119.

23. M. Spada and $\mathrm{H}$. Wobig, On the existence and uniqueness of dissipative plasma equilibria in a toroidal domain, J. Phys. A: Math. Gen., 25 (1992) 1575-1591.

24. G. Ströhmer, About an initial-boundary value problem from magnetohydrodynamics, Math. Z. 209 (1992) 345-362.

25. C. Sulem, Quelques résultats de régularité pour les équations de la MHD, C.R.A.S. Série A t. 285 (1977) 365-385.

26. H.S.G. Swann, The convergence with vanishing viscosity of nonstationary NavierStokes flow to ideal flow in $R^{3}$, Trans. Amer. Math. Soc. 157 (1971) 373-397.

27. M.E. Taylor, Partial Differential Equations I: Basic Theory (Springer-Verlag, 1996).

28. M. Sermange and R. Temam, Some Mathematical Questions Related to the MHD Equations, Comm. Pure. and Appl. Math. XXXVI (1983) 635-664.

29. R. Temam, Navier-Stokes Equations, Theory and Numerical Analysis (North-Holland, Amsterdam, 1979).

30. I. Vrabie, Semigrupuri de operatori liniari si aplicatii (Ed. "Universitatii Al. I. Cuza", Rumania, 2001).

31. J. Wu, Viscous and inviscid magneto-hidrodynamics equations, Journal Anal. Math. 73 (1997) 251-265. 